Garry Robins - Doing Social Network Research. Network-based Research Design for Social Scientists

\title{
Amandine Thiry
}

Émulations - Revue de sciences sociales

2017, «Comptes rendus critiques, En ligne »

\section{Article disponible à l'adresse suivante}

https://ojs.uclouvain.be/index.php/emulations/article/view/6893

\section{Pour citer cet article}

Amandine Thiry, « Garry Robins — Doing Social Network Research. Network-based Research Design for Social Scientists », Émulations, en ligne. Mise en ligne le 15 mai 2017. DOI : 10.14428/emulations.cr.002

Distribution électronique : Université catholique de Louvain (Belgique) : ojs.uclouvain.be

(C) Cet article est mis à disposition selon les termes de la Licence Creative Commons Attribution, Pas d'Utilisation Commerciale 4.0 International. http://creativecommons.org/licenses/by-nc/4.0/

Éditeur : Émulations - Revue de sciences sociales / Presses universitaires de Louvain https://ojs.uclouvain.be/index.php/emulations

ISSN électronique : 1784-5734

UCL PRESSES

UNIVERSITAIRES 


\title{
Garry Robins - Doing Social Network Research. Network-based Research Design for Social Scientists
}

\begin{abstract}
Amandine Thiry ${ }^{1}$
Recensé : Garry Robins, Doing Social Network Research. Network-based Research Design for Social Scientists, London, SAGE Publications Ltd, 2015, 264 p.

Récompensé par le Prix Simmel en 2016, Garry Robins appartient à une écurie chevronnée de chercheurs spécialistes en analyse de réseaux ; celle qui compose notamment le comité directeur de l'INSNA, l'International Network for Social Network Analysis, dont il est membre depuis 2007. Professeur au département de psychologie de l'Université de Melbourne, lauréat du Prix Linton Freeman pour sa « contribution systématique et substantielle » à l'étude scientifique des structures sociales ${ }^{2}$, il signe ici un manuel stimulant à plusieurs égards. Comme en témoigne le titre, l'ouvrage se concentre avant tout sur la conception d'une recherche en analyse de réseaux ${ }^{3}$, et non sur l'ensemble des méthodes statistiques communément désigné sous le nom de Social Network Analysis (SNA). La différence est de taille, le manuel se distingue des classiques du genre (Faust \& Wasserman, 1994 ; Scott, 2000) tout en se rapprochant de manuels plus récents (Henning, Brandes \& Mergel, 2012). Robins pose d'emblée l'intentionnalité des acteurs comme importante caractéristique de l'étude des réseaux sociaux par rapport à celle des réseaux au sens large (tendant à se regrouper sous la coupole de Network science ${ }^{4}$ ). Il s'agit donc de l'étude du réseau comme ontologie incluant à la fois des acteurs sociaux et des relations qu'ils possèdent entre eux (p. 5), définition certes classique (Faust \& Wasserman, 1994) mais indispensable au lecteur. L'objectif spécifique du manuel est de guider le chercheur en sciences sociales désirant s'initier
\end{abstract}

\footnotetext{
${ }^{1}$ Doctorante au sein du Centre d'histoire du droit et de la justice (UCL) et du groupe de recherche Sociale Geschiedenis na 1750 (UGent).

${ }^{2}$ INSNA, Freeman Award. En ligne, consulté le 11 novembre 2016. URL : http://insna.org/freeman.html.

${ }^{3}$ La locution « analyse de réseaux » est préférée à celle d'« analyse de réseaux sociaux » dans ce compte-rendu, car il s'agit du terme consacré en français. L'épithète "sociaux» serait pourtant souhaitable pour marquer l'importance de l'intentionnalité des acteurs que l'auteur souligne dans son manuel (cf. infra).
}

4 Cf. la revue éponyme, lancée en 2013, et l'article de Brandes e. a., 2013. 
à l'analyse de réseaux, de l'élaboration de ses questions de recherche à l'interprétation de ses résultats. L'objectif général — non moins explicite — est lui apprendre à penser "réseaux »: l'analyse de réseaux n'est pas, pour Robins, une méthode additionnelle dans la boîte à outils du chercheur, mais une perspective spécifique sur les réalités sociales, nécessitant au préalable d'assumer des prérequis théoriques et méthodologiques $^{5}$.

Dans le premier chapitre, l'auteur introduit les présupposés théoriques qu'implique l'analyse de réseaux, analysant ses spécificités par rapport à d'autres approches en sciences sociales. L'analyse de réseaux répond à un questionnement spécifique sur les structures du social et sur les processus qui les transforment. L'auteur met en garde le lecteur novice, listant quelques raisons jugées à elles seules insuffisantes pour introduire l'analyse de réseaux dans un travail de recherche. De façon didactique, Robins aborde ensuite les notions fondamentales et les questionnements qui sous-tendent la démarche (nœud, relation, degré, distribution, centralité, position dans le réseau, structure du réseau, réciprocité des relations, etc.). On soulignera d'emblée la clarté des exemples présentés. Pour débroussailler l'immense futaie de travaux relatifs à cette discipline en formation, Robins s'appuie en premier lieu sur des recherches qu'il connaît de près pour y avoir été personnellement impliqué (par exemple : Robins \& Kashima, 2008). Ce choix délibéré s'apprécie surtout pour la clarté avec laquelle l'auteur développe les exemples de ce premier chapitre, qui parcourront ensuite tout le livre au sein des encadrés « Pulling back the curtain ».

Les notions fondamentales de l'analyse de réseaux, qui permettent de dépasser l'usage métaphorique du mot « réseau », sont au cœur du second chapitre. Affinant leurs contours, ce chapitre définit précisément les concepts déterminants en les répartissant en cinq paragraphes thématiques. Quinze « principes » théoriques (liens forts et faibles, trous structuraux, multiplexité...) sont ensuite examinés tour à tour, accompagnés chacun d'un renvoi à une recherche récente. Robins veille à introduire la complexité en douceur dans ce second chapitre, usant de la notation mathématique avec didactisme et parcimonie.

Ces prémices théoriques posées, l'ouvrage adopte ensuite une structure qui suit au plus près le cheminement du chercheur en analyse de réseaux, contribuant ainsi à l'originalité de ce manuel. Le troisième chapitre, consacré à l'élaboration des questions de recherche, convoque les composantes (« building blocks ») d’une étude de réseaux sous forme de huit questions : Qui ou que sont les acteurs sociaux de l'analyse ? Quelles sont les limites du réseau ? Combien de types d'acteurs et quels types de liens relationnels sont à considérer? Quels sont les attributs des acteurs sociaux ? S'agit-il d'une étude diachronique ou synchronique du réseau ? etc. De la définition des acteurs à celle des

5 «A network perspective is not just a methodological extension to standard social science research, but carries theoretical commitments». (p. 14). 
liens relationnels, de la délimitation du réseau lui-même aux facteurs exogènes à prendre en compte dans l'analyse, les réponses à ces questions fondamentales détermineront la conception de l'étude à mener. C'est à ces différents choix posés par le chercheur que s'attaque Robins dans la seconde partie du chapitre, en mesurant leurs implications respectives sur la recherche ("strenghs », « issues », « examples »). Les historiens - communauté en expansion parmi les chercheurs en analyse de réseaux — pourront toutefois regretter qu'à la question « Is time relevant? », Robins n'ait répondu qu'en trois paragraphes visant à décourager les études longitudinales (ou diachroniques) vu le travail éreintant de la collecte des données ; à moins de disposer de «ressources abondantes, du soutien d'assistants de recherche, de temps, de répondants coopératifs et de compétences avancées en analyse de réseaux ${ }^{6} »$ (p.50).

Le chapitre 4, «Social systems and data structures: Relational ties and actor attributes », constitue à la fois un prolongement du chapitre 3 et un préalable méthodologique aux deux chapitres suivants. La conceptualisation du réseau doit s'accompagner d'une réflexion sur la structuration des données ou, plus précisément, sur le choix de la structure qui recueillera l'information relationnelle collectée. Qu'il soit " complet " ou « égocentrique », « multiplex » ou procède d'un échantillonnage en boule de neige, le réseau étudié et sa structure doivent être définis et considérés selon leur nature spécifique. Par deux chapitres complémentaires (5 et 6), Robins aborde ensuite les réflexions préalables, les obstacles et les choix méthodologiques que suppose la collecte des données (différentes formes de collectes, diversité des contextes de recherche, échantillonnage, fiabilité et validité des mesures, etc.). Ces deux chapitres font la part belle aux exemples et aux recommandations détaillées. On soulignera la diversité des contextes de collecte de données qu'aborde l'auteur dans le chapitre 6 , et surtout, l'utile bibliographie sélective commentée.

Après un septième chapitre très bref portant sur les problèmes éthiques liés à la collecte des données et à la diffusion des résultats (anonymat et confidentialité, consentement éclairé, devoir de prudence, feedback aux participants, etc.), Robins consacre le huitième chapitre à la représentation graphique. Il y souligne les limites des visualisations comme outils analytiques, tout en pointant leurs vertus pour l'exploration et la présentation des données. Ce chapitre vise avant tout le lecteur débutant, auquel sont livrés quelques clefs et principes généraux pour obtenir des visualisations de qualité. Pour clore ce diptyque sur l'analyse des données, l'auteur passe en revue dans le chapitre 9 les différentes méthodes communément rassemblées sous le terme Social Network Analysis (SNA). À l'exhaustivité est ici préféré un aperçu de différentes méthodes établies. L'auteur décortique la façon dont elles sont appliquées dans des recherches

\footnotetext{
${ }^{6}$ Traduction que nous effectuons à partir de la phrase suivante : "Of course, if you have plentiful resources, a good supply of research assistants, time, cooperative respondents and sophisticated network analytic skills, then - in the best of all possible worlds - collect data longitudinally and collect plenty of it. »
} 
récentes. Bien que l'analyse de réseaux binaires y tienne une place de choix, le dense chapitre brasse un très large spectre de méthodes, évitant la sophistication inutile malgré la complexité des notions traitées.

«Inférence, généralisation, causalité et autres sujets importants (weighty matters) » sont au centre du dixième et dernier chapitre, consacré à la manière de tirer des conclusions à partir d'une analyse de réseaux. Robins y développe une réflexion stimulante, bien que dense, sur l'interprétation et la généralisation des données, dépassant ainsi largement le cadre de l'analyse de réseaux. Ce dernier chapitre constitue un important plaidoyer pour une recherche en sciences sociales plus consciente des dangers de l'inférence et de la généralisation des résultats. À partir du cas particulier d'une étude controversée sur la diffusion de l'obésité par les réseaux amicaux (Christakis \& Fowler 2007), Robins argumente en faveur d'une inférence qu'il qualifie de scientifique, c'est-à-dire prenant en compte un ensemble de travaux et de nombreux facteurs, et non simplement de statistique.

En conclusion, le point fort de l'ouvrage de Robins est avant tout de parvenir à articuler analyse de réseaux et autres théories issues des sciences sociales, tout en renvoyant à des recherches concrètes et récentes. Malgré tout, le manuel prend régulièrement le point de vue d'un analyste de réseaux «pur-sang » et tente d'inciter le lecteur à penser "réseaux », au détriment parfois d'un débat critique à propos d'approches radicalement différentes. Ainsi, la théorie de l'« acteur-réseau » ${ }^{7}$ n'est mentionnée à aucun endroit de ce manuel (l'intentionnalité des acteurs ayant certes été portée comme principe-clef au début du premier chapitre). On regrettera encore quelques choix d'édition - l'absence d'une table des matières détaillée, un glossaire faisant parfois doublon avec le texte (p. 28-29) - et le caractère très incomplet de la bibliographie concernant les logiciels (notamment pour le logiciel Gephi). Si le livre s'adresse avant tout à un chercheur novice en analyse de réseaux, le caractère pointu des derniers chapitres séduira également le chercheur plus aguerri. Rédigé dans un style fluide et plutôt direct (« Do not be fooled.», «If another network expert tells you of other possibilities, listen. »), l'ouvrage tranche avec le ton plus neutre généralement adopté dans les manuels. Outre la qualité du dispositif didactique mis à la disposition du lecteur (glossaires, exemples, résumés et lectures conseillées en fin de chapitre), la diversité du bagage de Garry Robins permet de recommander la lecture de ce manuel à tout chercheur en sciences humaines intéressé par cette «network science » en voie de formation.

${ }^{7}$ Actor Network Theory (ANT), théorie développée notamment par Bruno Latour, permettant d'incorporer dans l'analyse des acteurs sans intentionnalité, tels des objets (Latour, 2005) 


\section{Bibliographie}

Henning M., Brandes U., Mergel I. (2012), Studying Social Networks: A Guide to Empirical Research, Frankfurt am Main, Campus Verlag.

LAtouR B. (2005), Reassembling the social. An introduction to Actor-Network Theory, Oxford, Oxford University Press; trad. Paris, La Découverte.

RoBINS G., Kashima Y. (2008), « Social psychology and social networks: Individuals and social systems », Asian Journal of Social Psychology, vol. 11, p. 1-12.

SCOTt J., CARRINGTON P. (dir.) (2011), The SAGE handbook of Social Network Analysis, London, SAGE.

Scotт J. (2013 [2000]), Social Network Analysis, Los Angeles, Sage.

Brandes U., Robins G., MCCRANIE A., WASSERMAN S. (2013), « What is network science? », Network Science, vol. 1, n 1, p. 1-15.

WASSERMAN S., FAUST K. (1994), Social network analysis: Methods and applications, Cambridge, Cambridge University Press. 